\title{
Long-Term Follow-Up of Children Treated With Peginterferon and Ribavirin for Hepatitis C Virus Infection
}

\author{
${ }^{*}$ Barbara Haber, ${ }^{\dagger}$ Estella Alonso, ${ }^{\ddagger}$ Alejandra Pedreira, ${ }^{\S}$ Norberto Rodriguez-Baez,

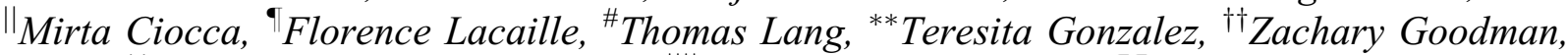 \\ ${ }^{\ddagger \ddagger}$ Zijiang Yang, ${ }^{\S \S}$ Beth Jackson, ${ }^{\prime \prime \mid}$ Stephanie Noviello, and ${ }^{\top \uparrow}$ Janice K. Albrecht
}

\begin{abstract}
Objectives: The aim of the study was to describe the 5-year follow-up of children who received peginterferon and ribavirin in a global, open-label study. Methods: A 5-year follow-up study of 107 children and adolescents ages 3 to 17 years with chronic hepatitis $\mathrm{C}$ virus infection who received peginterferon and ribavirin for 24 or 48 weeks. No drugs were administered during follow-up. Results: Ninety-four patients were enrolled in the long-term follow-up portion of the study; the median duration of follow-up was 287 weeks (range, 73-339). Of 63 patients with sustained virologic response who were enrolled, 54 completed 5 years of follow-up; none had relapse in the 5-year follow-up period. Significant decreases in height $z$ scores were observed during treatment. The effect of treatment on height $z$ score was larger in patients treated for 48 weeks compared with those treated for 24 weeks (mean change from baseline to the end of treatment was $-0.13[P<0.001]$ and -0.44 $[P<0.001]$ in the $24-$ and 48 -week treatment groups, respectively). Among patients treated for 24 weeks, full recovery of height $z$ scores to baseline was observed by 1 year of follow-up, whereas only partial recovery was observed during 5 years of follow-up in patients treated for 48 weeks (mean change from baseline to the final follow-up visit was $-0.16(P=\mathrm{NS})$ and $-0.32(P<0.05)$ in the 24- and 48-week treatment groups, respectively). Similar patterns were observed for weight and body mass index $z$ scores.

Conclusions: Impairment of growth should be considered when assessing the risk-benefit profile of peginterferon/ribavirin therapy in children with hepatitis $\mathrm{C}$ virus infection. In deciding to treat children with chronic hepatitis $\mathrm{C}$ virus, considerations should include both deferring treatment in patients during optimal growth periods, and the possibility that interferonfree regimens may be available to children in the next 5 to 10 years.
\end{abstract}

Key Words: growth, interferon, observational, pediatric

(JPGN 2017;64: 89-94)

Received September 16, 2015; accepted April 11, 2016.

From the *Clinical Research Department, Merck Sharp \& Dohme Corp., Kenilworth, NJ, the †Ann \& Robert H. Lurie Children's Hospital of Chicago, Chicago, IL, ‡Hepatologia Infantil, El Hospital de Niños Ricardo Gutierrez, Buenos Aires, Argentina, the §University of Texas Southwestern Medical Center, Dallas, TX, the $\mid$ Pediatric Hepatology and Liver Transplant Section, German Hospital, Buenos Aires, Argentina, the - Service de Gastroentérologie-Hépatologie-Nutrition pédiatriques, Hôpital Necker-Enfants Malades, Paris, France, the ${ }^{\#}$ Chefarzt der Klinik für Kinder und Jugendmedizin, Kindergastroenterologe, Neonatologe, Klinikum Starnberg, Akademisches Lehrkrankenhaus der Ludwig-Maximilians-Universität, München, Germany, the ${ }^{*}$ Hospital de Niños Sor Maria Ludovica de La Plata, La Plata, Argentina, the ††Center for Liver Diseases, Inova Fairfax Hospital, Falls Church, VA, the $\ddagger$ Chesterbrook, PA, §§Merck \& Co., Inc., North Wales, PA, and the || $\mid$ |Bristol-Myers Squibb Company, Princeton, NJ, and $\uparrow \uparrow$ Winter Park, FL.

Address correspondence and reprint requests to Barbara Haber, MD, Senior Principal Scientist, Hepatology, Infectious Disease, Clinical Research Department, Merck Sharp \& Dohme Corp., 351 North Sumneytown Pike, Office UG3D02, North Wales, PA 19454

(e-mail: barbara.haber@merck.com)

Supplemental digital content is available for this article. Direct URL citations

\section{What Is Known}

- Rates of sustained virologic response are approximately $58 \%$ in children with hepatitis $C$ virus genotype (G) 1 infection treated for 48 weeks.

- Growth inhibition occurs in children during treatment with peginterferon and ribavirin.

\section{What Is New}

- Impaired growth is largely recovered posttreatment in children who received peginterferon/ribavirin for 24 weeks, but only partial recovery is seen in children treated for 48 weeks.

$t$ is unclear whether benefits outweigh the risks when considering treatment of chronic hepatitis $\mathrm{C}$ virus ( $\mathrm{HCV}$ ) infection during childhood. Although the progressive nature of HCV infection is considered to be slower in children than in adults, it remains a chronic liver condition. If left unchecked, HCV can result in hepatic fibrosis, and ultimately decompensation or hepatocellular carcinoma. Data suggest that up to $5 \%$ of infected children may develop severe hepatic fibrosis or cirrhosis by the time they reach adulthood (1). Delaying treatment may, therefore, be associated with irreversible liver disease in a small proportion of children (1).

appear in the printed text, and links to the digital files are provided in the HTML text of this article on the journal's Web site (www.jpgn.org). www.clinicaltrials.gov registration number: NCT00104052.

The study was funded by Merck \& Co., Inc.

B.H. is an employee of Merck Sharp \& Dohme Corp. and owns stock in Merck \& Co., Inc. E.A. has received travel support from Merck \& Co., Inc., and her institution has received research grants from Merck \& Co., Inc. T.L. is a consultant for Astellas and has been on a speaker's bureau for AbbVie.Z.G.'s institution has received research grants from Merck \& Co., Inc., Gilead Sciences, Tobira, Galacta Therapeutica, Intercept, Excellenze, Conatus, and Synageva. Z.Y. and J.K.A. are former employees of Merck \& Co., Inc. B.J. is an employee of and owns stock in Merck \& Co., Inc. S.N. was a consultant for Schering-Plough, was a former employee of Schering-Plough and BristolMyers Squibb, received payment for manuscript preparation from ScheringPlough, and owns stock in Merck \& Co., Inc., Bristol-Myers Squibb, and Johnson \& Johnson. The remaining authors report no conflicts of interest. Copyright $(\mathcal{C} 2016$ by European Society for Pediatric Gastroenterology, Hepatology, and Nutrition and North American Society for Pediatric Gastroenterology, Hepatology, and Nutrition DOI: $10.1097 / M P G .0000000000001239$ 
Furthermore, children with HCV infection have a communicable disease that can result in exclusion from activities such as sports; it is also preferable to treat $\mathrm{HCV}$ infection before a person becomes sexually active or becomes pregnant.

Treatment options for children with hepatitis $\mathrm{C}$ are limited. Direct-acting antiviral agents have dramatically altered the HCV treatment landscape in adult patients, but these agents are not yet approved for use in children. Therefore, peginterferon and ribavirin remains the standard of care for children with $\mathrm{HCV}$ infection involving a lengthy treatment regimen associated with unfavorable tolerability, and without guaranteed viral eradication (2-4). Rates of sustained virologic response (SVR) are approximately $58 \%$ in children with HCV treated with peginterferon and ribavirin (3).

The risks of progressive liver disease and the challenging treatment regimen must be weighed against the opportunity for viral eradication when considering treatment of children with $\mathrm{HCV}$ infection. One of the major risks regarding treatment of children with interferon-based therapy is the potential effect on growth and development. Reduced growth rates in children have been reported during interferon-based therapy, and it remains unclear to what extent growth is recoverable after completion of treatment $(2,5,6)$. Wirth and colleagues studied the treatment of HCV infection in 107 children receiving peginterferon and ribavirin in a global, openlabel study (2). Weight loss was common but was largely recovered during the 24-week follow-up period; mean weight percentile was 56.6 at baseline and 53.4 at end of follow-up. Linear growth was also inhibited during treatment and remained below the median for the US population at the end of 24 weeks of follow-up (mean height percentiles were 50.9 at baseline and 44.25 at the end of follow-up). Similarly, Jonas et al (6) reported declining weight, height, and body mass index (BMI) scores in children receiving peginterferon with or without ribavirin in the Pediatric Study of Hepatitis C (PEDS-C). In the present study, weight-for-age and BMI-forage scores returned to baseline values after completion of treatment; however, height-for-age scores had not recovered to baseline 96 weeks after treatment completion (6). Here, we report on the 5 -year follow-up of the children and adolescents enrolled in the study by Wirth et al (2). Children who attained SVR were followed for durability of response, and the long-term implications of interferon-based therapy, particularly with regard to height and weight development, were also studied.

\section{METHODS}

This is a long-term follow-up (LTFU) study of children with $\mathrm{HCV}$ infection enrolled in a global, multicenter, open-label study to evaluate the efficacy, safety, and pharmacokinetics of peginterferon alfa-2b and ribavirin (NCT00104052; protocol P02538). Full details of the study have been published previously (2). Informed consent was obtained from all patients before enrollment in the initial treatment study and again when patients entered the follow-up protocol. These studies were conducted in accordance with the provisions of the Declaration of Helsinki, the International Conference on Harmonization guidelines, and other regulations governing clinical study conduct. The protocols were approved by an independent ethics committee or institutional review board at each participating site.

\section{Patients and Initial Treatment}

Children ages 3 to 17 years with previously untreated $\mathrm{HCV}$ infection were eligible for enrollment in the original treatment study. Children with decompensated liver disease, human immunodeficiency virus or hepatitis B virus coinfection, or other significant comorbidities were excluded from the original treatment protocol.
All patients received peginterferon alfa- $2 \mathrm{~b}\left(60 \mu \mathrm{g} / \mathrm{m}^{2} /\right.$ week, based on approximate equivalence to the adult-approved dose of $1.5 \mu \mathrm{g} / \mathrm{kg} / \mathrm{week})$ and ribavirin $(15 \mathrm{mg} / \mathrm{kg} /$ day, based on previously established effective dosing (7)). Doses of ribavirin did not exceed $1200 \mathrm{mg} /$ day; therefore, children with body weight $>90 \mathrm{~kg}$ were also excluded. Patients with HCV G1, G2, or G3, and baseline viral load $\geq 600,000 \mathrm{IU} / \mathrm{mL}$ were treated for 48 weeks, and those with G2 or G3 infection and low baseline viral load $<600,000 \mathrm{IU} / \mathrm{mL}$ were treated for 24 weeks. Patients with $<2 \log _{10}$ decline in $\mathrm{HCV}$ RNA at week 12 or detectable HCV RNA at week 24 were discontinued from the study based on futility. The primary end point in the original treatment protocol was SVR (defined as undetectable HCV RNA at follow-up week 24). Secondary end points were on-treatment virologic response and safety assessments, including measurements of weight and height.

\section{Long-Term Follow-up}

Patients who received $\geq 1$ dose of medication during the initial treatment study and completed 24 weeks of posttreatment follow-up were eligible for enrollment in the LTFU portion of the study. No drugs were administered during the LTFU portion. Patients who underwent re-treatment for $\mathrm{HCV}$ infection after completing the initial treatment protocol were excluded from the follow-up study.

Initial follow-up visits were scheduled approximately 1 year after the follow-up week 24 visit in the original study. Patients who attained SVR had 1 visit per year for up to 5 years, whereas patients with detectable HCV RNA at follow-up week 24 (no SVR) had visits every 6 months during follow-up. Patients who were not able to enter the study at the year 1 visit were able to enter the study at later time points.

The primary end point of this follow-up study was durability of SVR. HCV RNA levels were measured yearly for assessment of durability of SVR and every 6 months in non-SVR patients. A patient was classified as a sustained responder at a given time point if HCV RNA was below the lower limit of quantitation (LLQ) at that time point. Plasma HCV RNA levels were measured at a central laboratory using the HCV RNA COBAS Taqman 2.0 assay (Roche, Indianapolis, IN, LLQ $=25 \mathrm{IU} / \mathrm{mL}$ ). Confirmatory testing was performed in patients with initial SVR and subsequent detectable HCV RNA during follow-up. If the initial positive value was confirmed, then HCV genotype was tested at the central laboratory to determine whether the patient had relapsed or had been reinfected with a different genotype.

Safety assessments, along with physical examinations (including a gastrointestinal/liver examination), vital signs, body weight, height, and laboratory safety tests (serum chemistry, hematology, thyroid-stimulating hormone [TSH]) were performed at each visit. Only serious adverse events (SAEs) that occurred after the patient had completed the 24-week posttreatment follow-up were reported. All adverse events relating to progression of liver disease were reported as SAEs.

Height was measured using the same instrument at each clinic visit (stadiometer, if available), or measuring tape affixed to the wall; weight was measured using the same scales at each clinic visit. Growth analyses were adjusted for age (height, weight, BMI percentiles), and $z$ scores were derived for each patient using an SAS macro provided by the Centers for Disease Control and Prevention. Each $z$ score represents data as a standard deviation (SD) from the population norm within each age group. For example, a height $z$ score of -1 indicates a value 1 SD below the normative population for height, whereas an $z$ score of +1 is 1 SD above the normative population. $Z$ scores were summarized using descriptive 
statistics as changes from baseline at each time point during follow-up, where baseline was taken as the start of the treatment period. An ad-hoc statistical test of the $z$ scores was performed by parameter and time point to test null hypothesis of $0 ; P$ values are 2 -sided, based on the $t$ distribution, and unadjusted for multiple comparisons.

\section{RESULTS}

\section{Patient Disposition and Baseline Demographics}

In total, 94 of the 107 patients who participated in the original treatment study across 20 sites in 7 countries were enrolled in this LTFU analysis (Table 1). The study disposition reflects that 80 patients completed the study and 14 discontinued before completing 5 years of follow-up (Supplementary Fig. 1, Supplemental Digital Content, http://links.lww.com/MPG/A664). Of the 14 patients who discontinued, 1 was due to noncompliance with the protocol, 6 were due to withdrawal of consent, 6 were lost to follow-up, and 1 was for administrative reasons. The median duration of follow-up for these 94 patients was 287 weeks (range, 73-339).

Of the 94 patients followed during LTFU, 48\% were boys, $52 \%$ were 3 to 10 years of age at enrolment, and $48 \%$ were 11 to 17 years of age. None of the patients had a medical history of thyroid disorders or insulin-dependent diabetes. Approximately half of the enrolled patients $(46 / 94,49 \%)$ were treated for $\sim 24$ weeks, of which $26(57 \%)$ had SVR and 20 (43\%) were nonresponders. The remaining 48 patients $(51 \%)$ were treated for $\sim 48$ weeks, of which $37(77 \%)$ had SVR and $11(23 \%)$ were nonresponders. One patient

\begin{tabular}{|c|c|c|c|}
\hline & \multicolumn{3}{|c|}{ Patients } \\
\hline & $\begin{array}{l}\text { Age } 3-10 y \\
(\mathrm{n}=49)\end{array}$ & $\begin{array}{l}\text { Age } 11-17 y \\
(n=45)\end{array}$ & $\begin{array}{c}\text { All } \\
(\mathrm{n}=94)\end{array}$ \\
\hline \multicolumn{4}{|l|}{ Sex } \\
\hline Male & 18 & 27 & 45 \\
\hline Female & 31 & 18 & 49 \\
\hline \multicolumn{4}{|l|}{ Race } \\
\hline White & 46 & 35 & 84 \\
\hline Black & 1 & 0 & 1 \\
\hline Asian & 1 & 5 & 6 \\
\hline Multiracial & 1 & 2 & 3 \\
\hline \multicolumn{4}{|l|}{ Ethnicity } \\
\hline Hispanic/Latino & 9 & 4 & 13 \\
\hline Non-Hispanic/Latino & 40 & 41 & 81 \\
\hline \multicolumn{4}{|l|}{ Genotype } \\
\hline G1 & 33 & 28 & 61 \\
\hline G2 & 6 & 9 & 15 \\
\hline G3 & 8 & 6 & 14 \\
\hline G4 & 2 & 2 & 4 \\
\hline \multicolumn{4}{|l|}{ Baseline viral load } \\
\hline$<600,000 \mathrm{IU} / \mathrm{mL}$ & 28 & 24 & 52 \\
\hline$>600,000 \mathrm{IU} / \mathrm{mL}$ & 19 & 19 & 38 \\
\hline Missing & 2 & 2 & 4 \\
\hline \multicolumn{4}{|c|}{ Previous treatment duration } \\
\hline 24 weeks & 20 & 26 & 46 \\
\hline 48 weeks & 29 & 19 & 48 \\
\hline \multicolumn{4}{|c|}{ Previous treatment outcome } \\
\hline SVR & 32 & 31 & 63 \\
\hline Nonresponse & 17 & 14 & 31 \\
\hline
\end{tabular}

$\mathrm{SVR}=$ sustained virologic response. in the original treatment study discontinued after 18 weeks of treatment.

\section{Durability of Sustained Virologic Response}

A total of 63 patients with SVR from the treatment portion of the protocol were enrolled, 54 of whom completed 5 years of follow-up (Supplementary Fig. 2, Supplemental Digital Content, http://links.lww.com/MPG/A665). All 54 patients had valid HCVRNA results at year 5 and none relapsed during the 5-year follow-up period (in all patients HCV RNA was below the LLQ of $25 \mathrm{IU} / \mathrm{mL}$ throughout follow-up).

Two patients had HCV-RNA levels that were detectable but below the LLQ during the LTFU. One of these patients had detectable/unquantifiable HCV RNA at the year 3 time point (confirmed on retesting of the same sample), and also had a similar result on a sample taken approximately 6 months later. The year 4 sample was not available for analysis; however, testing of the year 5 sample from this patient revealed undetectable HCV RNA. The second patient with unquantifiable HCV RNA during follow-up had detectable/unquantifiable HCV RNA at year 5 and was not monitored further. Genotype data from samples obtained during followup are not available for either patient.

Of the 9 patients with SVR who did not complete 5 years of follow-up, 7 dropped out prematurely (none of whom had relapsed before discontinuation) and 2 patients completed 5 years of followup with sustained response through LTFU year 4 , but the year 5 sample was not available for analysis.

There were 31 previous nonresponders with quantifiable HCV RNA at the end of the original treatment protocol who entered the follow-up study. None had HCV RNA <LLQ during the follow-up period.

\section{Safety}

A total of 5 SAEs were reported in 3 patients during LTFU, none of which were considered to be related to peginterferon/ ribavirin therapy. Two patients had appendicitis, and there were 3 reports of talipes (growth delay of congenital clubfoot) in 1 patient. There were no deaths, no discontinuations due to an adverse event, and no discontinuations due to chronic liver disease.

\section{Height}

Significant decreases in height $z$ scores were observed during treatment. The effect of treatment on mean change from baseline in height $z$ score was larger in patients treated for 48 weeks compared with those treated for 24 weeks. Patients treated for 24 weeks had a mean change from baseline in height $z$ score of $-0.13(\mathrm{SD}=0.20$, $P<0.001)$ at the end of treatment, and $-0.16(\mathrm{SD}=0.62, P=\mathrm{NS})$ at the end of follow-up. Among patients treated for 24 weeks, mean height $z$ scores had returned to baseline levels by year 1 of follow-up and remained stable thereafter. In contrast, patients treated for 48 weeks had a mean change from baseline in height $z$ score of $-0.44(\mathrm{SD}=0.34, P<0.001)$ at the end of treatment and $-0.32(\mathrm{SD}=0.80, P<0.05)$ at the end of follow-up. Mean height $z$ scores declined during the treatment period, partially recovered by year 1 of follow-up, but then showed no further increase with continued follow-up (Table 2, Fig. 1).

The mean change in height percentile from baseline to the final follow-up visit was -3.0 percentiles and -8.9 percentiles for the 24- and 48-week treatment groups, respectively. Supplementary Table 1 (Supplemental Digital Content, http://links.lww.com/MPG/ A666) presents data according to sex and age subgroup. Overall, 
patient numbers are small, and effect on mean height percentile appears to be primarily related to treatment duration (24 vs 48 weeks), regardless of age or sex.

Overall, 30 of 94 (31.9\%) of patients had a decrease in height of $>15$ percentiles between baseline in the original treatment protocol and the final follow-up visit. The proportion of patients with a reduction of $>15$ percentiles was similar in boys and girls (31\% vs $33 \%$ ), but appeared higher in the 48 -week treatment group compared with the 24 -week treatment group. In total, 12 of $30(40 \%)$ girls and 7 of $18(39 \%)$ boys who received 48 weeks of treatment had a $>15$ percentile-point decrease in height from baseline to the end of follow-up, whereas 4 of 19 (21\%) girls and 7 of $27(26 \%)$ boys treated for 24 weeks had a $>15$ percentile-point decrease in height from baseline to the end of follow-up.

\section{Weight and Body Mass Index}

Weight $z$ scores decreased during treatment, with a mean change from baseline of -0.50 and -0.75 at the end of treatment in patients treated for 24 and 48 weeks, respectively. There was then a marked rebound during year 1 of follow-up, with a mean change from baseline in weight $z$ scores of +0.03 and -0.16 at the end of year 1 in patients treated for 24 and 48 weeks, respectively, and stabilizing thereafter for the remainder of follow-up (mean change from baseline in weight $z$ scores at the end of LTFU were -0.08 and -0.21 , respectively; Table 3, Fig. 1). Similar profiles were also seen with mean change from baseline in BMI $z$ scores during treatment and follow-up (Table 3, Fig. 1).

\section{Thyroid Dysfunction and Insulin-Dependent Diabetes}

Abnormal TSH levels were measured in 22 of 94 (23\%) patients during treatment or follow-up. During the treatment phase, $20(21 \%)$ of the patients had abnormal TSH values, most of which were above the normal range. During follow-up, 4 (4\%) of the patients had an abnormal TSH level: 2 had abnormal TSH levels during treatment and during follow-up, 1 had an abnormal TSH level during year 1 of follow-up with normal values throughout the treatment phase, and 1 had an abnormal TSH level at the final year 5 follow-up visit.

Three patients received levothyroxine during the treatment phase and 1 patient was treated during the LTFU portion: 3 had subsequent normal TSH values throughout LTFU; 1 had normal TSH values at LTFU years 1 and 2 and an elevated TSH level at LTFU year 3. No patient developed insulin-dependent diabetes.

\section{DISCUSSION}

Data from the present analysis further inform the debate regarding treatment of children with $\mathrm{HCV}$ with interferon-based antiviral therapy. In our cohort, SVR was durable with no late relapse and, therefore, consistent with previous reports of LTFU in children $(5,8)$ and with observations in adult patients $(9-11)$. SVR can be considered to be indicative of viral eradication.

The present study represents a long-duration follow-up of children treated for HCV with interferon-based antiviral therapy. Importantly, a relatively low rate of thyroid disease was observed following treatment, with abnormal TSH levels reported in only $4 \%$ of patients during LTFU. We, however, found that treatment with peginterferon and ribavirin was associated with impaired growth. During the treatment portion of the protocol, height, weight, and BMI $z$ scores declined compared with a normative population by $-0.13,-0.50$, and -0.60 , respectively, for those treated for $<48$ weeks and by $-0.44,-0.73$, and -0.71 , respectively, for those treated for $\geq 48$ weeks. In children treated for $<48$ weeks, a full recovery to pretreatment $z$ score was observed for height, weight, and BMI by 1 year after the end of treatment. For children treated for $\geq 48$ weeks, height, weight, and BMI demonstrated some recovery toward pretreatment $z$ score at 2 years of follow-up; however, full recovery for height, weight, or BMI was still not achieved at follow-up year 5 .

In this analysis, height was measured using the same instrument at each clinic visit (stadiometer, if available), or measuring tape affixed to the wall, and weight was measured using the same scales at each clinic visit. Although Tanner staging was performed during this study, the effect of treatment duration on height as it relates to sexual maturation could not be evaluated because of small sample sizes when data were split by Tanner stage, sex, and duration of treatment. We also do not have a full assessment of parental height as it relates to growth during the study. Parental height data for both parents are only available for 14 of 30 patients with a $>15$ percentile decrease in height, of which 10 patients had an estimated final height within the normal range.

A number of other studies have reported a similar effect of interferon-based antiviral therapy on growth in children $(2,4,7)$. A meta-analysis of 8 studies assessing interferon-based antiviral therapy in children with $\mathrm{HCV}$ infection noted small growth inhibition, although data were generally considered inconsistent and unsuitable for meta-analytical methods (3). Overall studies with native interferon tend to report a reversible effect on growth inhibition, with a decrease in height or weight percentile during treatment that is matched by an increase in percentile during the 24-week follow-up, even when treatment is continued for up to 48 weeks (7). In contrast, studies with pegylated interferon indicate a more profound inhibition of growth during treatment that is not fully recovered during the 24-week follow-up period $(2,6)$.

Fewer investigations have considered the long-term consequences of treatment-related growth impairment beyond the standard 24-week follow-up period that is typically used in the treatment of HCV infection. Follow-up of children enrolled in

TABLE 2. Mean change from baseline in height $z$ score, adjusted for baseline age and sex

\begin{tabular}{lccccccc}
\hline & EOT & $\begin{array}{c}\text { 24 weeks } \\
\text { follow-up }\end{array}$ & $\begin{array}{c}\text { LTFU } \\
\text { year 1 }\end{array}$ & $\begin{array}{c}\text { LTFU } \\
\text { year 2 }\end{array}$ & $\begin{array}{c}\text { LTFU } \\
\text { year 3 }\end{array}$ & $\begin{array}{c}\text { LTFU } \\
\text { year 4 }\end{array}$ & $\begin{array}{c}\text { LTFU } \\
\text { year 5 }\end{array}$ \\
\hline $\begin{array}{c}\text { Peginterferon/ribavirin } \\
\text { for 24 wk (n=46) }\end{array}$ & $-0.13^{*}(0.20)$ & $-0.12^{* *}(0.29)$ & $-0.04(0.43)$ & $+0.08(0.55)$ & $0(0.57)$ & $+0.06(0.56)$ & $-0.16(0.62)$ \\
$\begin{array}{c}\text { Peginterferon/ribavirin } \\
\text { for 48 wk (n=48) }\end{array}$ & $-0.44^{*}(0.34)$ & $-0.41^{*}(0.36)$ & $-0.32^{*}(0.53)$ & $-0.21^{* * *}(0.60)$ & $-0.25^{* * *}(0.62)$ & $-0.21(0.76)$ & $-0.32^{* * *}(0.80)$ \\
\hline
\end{tabular}

EOT $=$ end of treatment; LTFU $=$ long-term follow-up.

${ }^{*} P<0.001$.

${ }^{* *} P<0.01$.

${ }^{* * *} P<0.05$. 

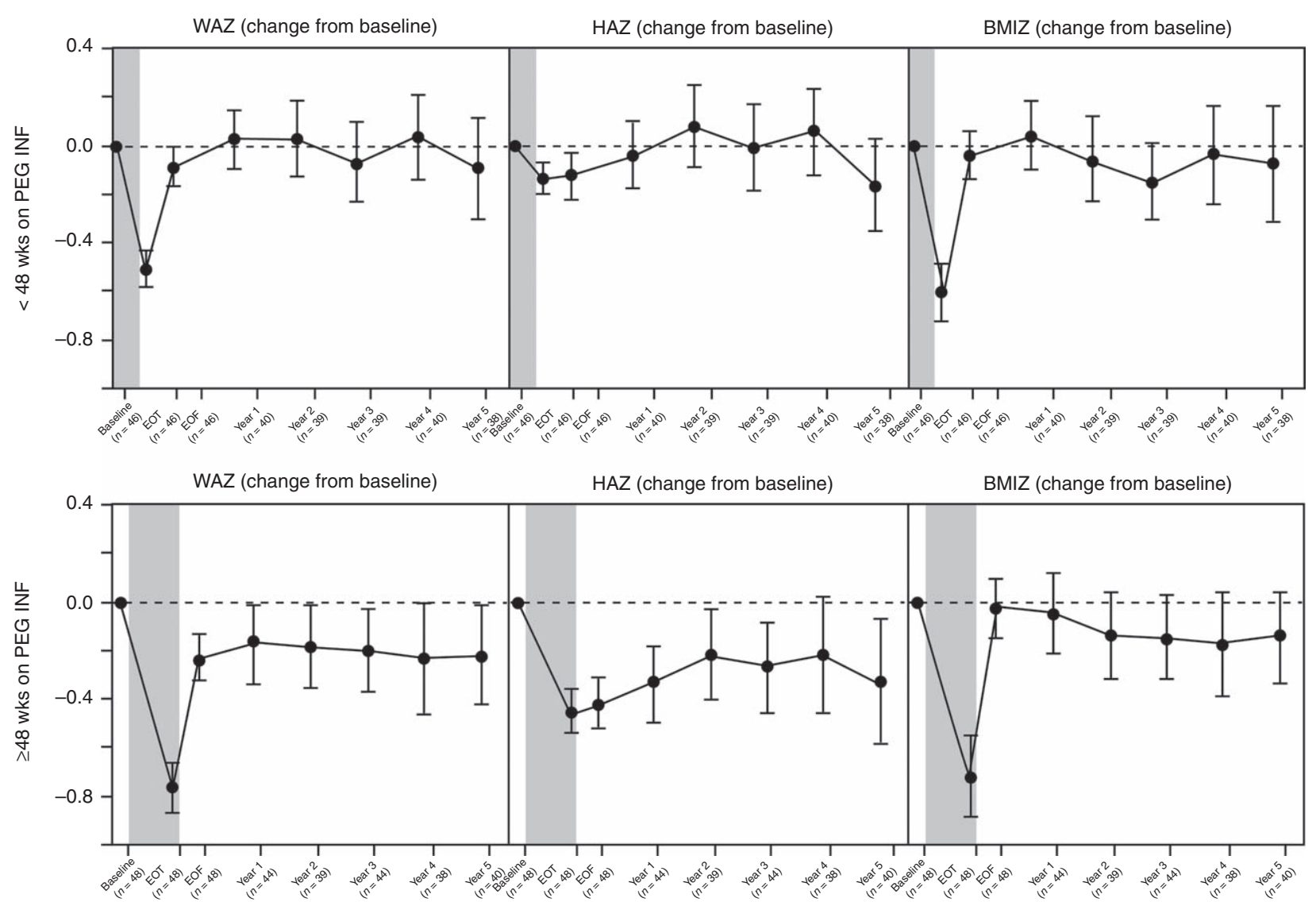

FIGURE 1. Change from baseline in $z$ scores for weight, height, and BMI. BMI = body mass index, BMIZ $=\mathrm{BMI}$-for-age $z$ score, EOF $=$ end of follow-up, EOT = end of treatment, HAZ = height-for-age $z$ score, PEG INF = pegylated interferon, WAZ = weight-for-age $z$ score.

PEDS-C showed significant growth inhibition in children treated with peginterferon and ribavirin when followed for up to 2 years, particularly among children treated for at least 48 weeks (6). Among a small subgroup of patients followed-up for up to 6 years after treatment, no long-term effects on height were identified that could be attributed to HCV treatment (8). Among 28 patients who received treatment for 48 to 52 weeks, $4(14 \%)$ had a $>15$-percentile point reduction in height at 2 years after treatment; at the end of LTFU, only $2(7 \%)$ remained $>15$-percentile points below baseline, one of whom had precocious puberty and the other genetic short stature based on height of family members (8). In contrast to the present study which enrolled $88 \%$ (94/107) of patients from the original treatment study, LTFU of the PEDS-C cohort included only a limited subset $(33 \%, 38 / 114)$ of patients from the original treatment study (8). Similarly, Kelly et al (5) reported growth inhibition in children treated with native interferon and ribavirin, with partial recovery in height percentile during LTFU. Among 97 children treated with interferon alfa and ribavirin $(70 \%$ of whom were followed for 5 years), mean height percentiles were 48 at pretreatment baseline, 37 at end of treatment week 24,40 at the 24-week posttreatment follow-up, and 44 at the final LTFU visit. Median duration of follow-up was 284 weeks (5). Furthermore, 21 of the patients followed by Kelly et al had a $>15$ percentile-point decrease in height at their final visit during LTFU and 14 of these 21 agreed to continued monitoring ( 9 patients had complete data; 5 patients could not be evaluated due to missing parental heights).

TABLE 3. Mean change from baseline in weight and body mass index $z$ scores, adjusted for baseline age and sex

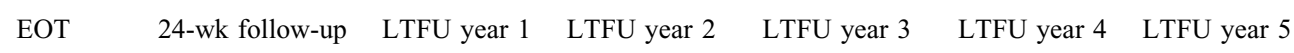

Weight

$\begin{array}{llllllll}\text { Peginterferon/ribavirin for 24 wk } & -0.50^{*}(0.26) & -0.08(0.27) & +0.03(0.41) & +0.04(0.52) & -0.06(0.53) & +0.04(0.56) & -0.08(0.68) \\ \text { Peginterferon/ribavirin for 48 wk } & -0.75^{*}(0.39) & -0.22^{*}(0.34) & -0.16(0.55) & -0.17(0.58) & -0.19^{* *}(0.59) & -0.22(0.73) & -0.21(0.71) \\ \text { BMI } & & & & & & & \\ \text { Peginterferon/ribavirin for 24 wk } & -0.60^{*}(0.41) & -0.04(0.34) & +0.04(0.48) & -0.05(0.58) & -0.14(0.53) & -0.04(0.66) & -0.07(0.73) \\ \text { Peginterferon/ribavirin for 48 wk } & -0.71^{*}(0.61) & -0.02(0.45) & -0.04(0.56) & -0.13(0.59) & -0.14(0.58) & -0.17(0.70) & -0.14(0.62)\end{array}$

$\mathrm{BMI}=$ body mass index; EOT $=$ end of treatment $\mathrm{LTFU}=$ long-term follow-up.

${ }^{*} P<0.001$.

${ }^{* *} P<0.05$. 
Three of 9 patients were younger than 20 years at continued followup and all 3 continued to show height deficits $>15$ percentile points. The remaining 6 patients were older than 20 years at final followup; only 1 patient had greater than the predicted midparental height, but all 6 patients had heights within 2 SDs of the predicted height. Thus, among these 14 children who had a $>15$ percentile-point decrease in height during treatment with peginterferon and ribavirin, growth impairment was not recovered when assessed as young adults. Overall, our data are consistent with those reported by Jonas et al and Kelly et al, suggesting that treatment with peginterferon and ribavirin is associated with growth inhibition. Our data, for the first time, reveal that growth inhibition during treatment is not recovered, even when children are followed for up to 5 years after completing therapy.

In children ages 3 to 17 years, peginterferon alfa- $2 b$, in combination with ribavirin, is approved for the treatment of chronic hepatitis $\mathrm{C}$ at a dose of $60 \mu \mathrm{g} / \mathrm{m}^{2} /$ week (12). Peginterferon alfa-2a is approved for children older than 5 years at a dose of $180 \mu \mathrm{g} / 1.73 \mathrm{~m}^{2} \times$ body surface area. The prescribing information for both peginterferon alfa- $2 \mathrm{a}$ and $-2 \mathrm{~b}$ suggest a treatment duration of 24 weeks in patients with G2/3 infection and 48 weeks for patients with other genotypes, and also note the potential for weight loss and growth impairment during treatment. Based on futility, patients with a $<2 \log _{10}$ decline in $\mathrm{HCV}$ RNA at treatment week 12 or detectable HCV RNA at treatment week 24 should be withdrawn from treatment (12). Based on the findings of this analysis, the following warning has been added to the peginterferon alfa-2b label: "Weight and height gain of pediatric patients lags behind that predicted by normative population data for the entire length of treatment. Severely inhibited growth velocity was observed in $70 \%$ of the patients while on treatment. Of these patients, $20 \%$ had continued inhibited growth velocity after 6 months of follow-up" (12). The most recent treatment guidelines from the American Association for the Study of Liver Diseases (AASLD) do not include recommendations for treatment of children with HCV infection (13); however, older guidelines note the approval of peginterferon alfa- $2 b$ and ribavirin in this indication (14). Interferon-free regimens using direct-acting antiviral agents, such as sofosbuvir and ledipasvir/sofosbuvir, are currently being investigated in children younger than 18 years (15), and future studies are planned for elbasvir/grazoprevir.

In conclusion, SVR was observed to be durable in the present study. All patients with SVR during the treatment phase who enrolled in the LTFU continued to be sustained responders. Among children treated for $<48$ weeks, height, weight, and BMI demonstrated full recovery to baseline values within 1 year of end of treatment. Only partial recovery was, however, seen in patients treated for $\geq 48$ weeks. These data suggest that impairment of growth should be considered when assessing the risk-benefit profile of peginterferon/ribavirin therapy in children with HCV infection. In deciding to treat children with chronic HCV, the risks and benefits should include both the timing of interferon-based therapy, and the possibility that interferon-free regimens may likely be available to children in the next 5 to 10 years.
Acknowledgments: The authors thank Tim Ibbotson, $\mathrm{PhD}$, and Beth McMahon-Wise, PhD, of ApotheCom, Yardley, PA for their contribution in medical writing and editorial assistance. The authors also acknowledge the contributions of Dr Flavia Bortolotti in the initial treatment study.

\section{REFERENCES}

1. Guido M, Bortolotti F, Leandro G, et al. Fibrosis in chronic hepatitis C acquired in infancy: is it only a matter of time? Am J Gastroenterol 2003;98:660-3.

2. Wirth S, Ribes-Koninckx C, Calzado MA, et al. High sustained virologic response rates in children with chronic hepatitis $\mathrm{C}$ receiving peginterferon alfa-2b plus ribavirin. J Hepatol 2010;52:501-7.

3. Druyts E, Thorlund K, Wu P, et al. Efficacy and safety of pegylated interferon alfa-2a or alfa-2b plus ribavirin for the treatment of chronic hepatitis $\mathrm{C}$ in children and adolescents: a systematic review and metaanalysis. Clin Infect Dis 2013;56:961-7.

4. Schwarz KB, Gonzalez-Peralta RP, Murray KF, et al. The combination of ribavirin and peginterferon is superior to peginterferon and placebo for children and adolescents with chronic hepatitis C. Gastroenterology 2011;140:450-8.

5. Kelly DA, Haber B, Gonzalez-Peralta RP, et al. Durability of sustained response shown in paediatric patients with chronic hepatitis $\mathrm{C}$ who were treated with interferon alfa-2b plus ribavirin. $J$ Viral Hepat 2012;19:263-70.

6. Jonas MM, Balistreri W, Gonzalez-Peralta RP, et al. Pegylated interferon for chronic hepatitis $\mathrm{C}$ in children affects growth and body composition: results from the pediatric study of hepatitis C (PEDSC) trial. Hepatology 2012;56:523-31.

7. Gonzalez-Peralta RP, Kelly DA, Haber B, et al. Interferon alfa-2b in combination with ribavirin for the treatment of chronic hepatitis $\mathrm{C}$ in children: efficacy, safety, and pharmacokinetics. Hepatology 2005;42:1010-8.

8. Jonas MM, Schwarz KB, Gonzalez-Peralta R, et al. Long-term growth outcomes in children treated for chronic hepatitis C. J Pediatr 2014;165:1252-4.

9. Swain MG, Lai MY, Shiffman ML, et al. A sustained virologic response is durable in patients with chronic hepatitis $\mathrm{C}$ treated with peginterferon alfa-2a and ribavirin. Gastroenterology 2010;139:1593-601.

10. Rutter K, Hofer H, Beinhardt S, et al. Durability of SVR in chronic hepatitis $\mathrm{C}$ patients treated with peginterferon-alpha2a/ribavirin in combination with a direct-acting anti-viral. Aliment Pharmacol Ther 2013;38:118-23.

11. Manns MP, Pockros PJ, Norkrans G, et al. Long-term clearance of hepatitis $\mathrm{C}$ virus following interferon alpha-2b or peginterferon alpha2b, alone or in combination with ribavirin. J Viral Hepat 2013;20:524-9.

12. US Prescribing Information (USPI). PegIntron 50 Micrograms Powder and Solvent for Solution for Injection. Whitehouse Station, NJ: Merck \& Co., Inc.; 2013.

13. American Association for the Study of Liver Diseases, Infectious Disease Society of North America. AASLD, IDSA, IAS-USA. Recommendations for testing, managing, and treating hepatitis C. AASLD/ IDSA Web Site. http://www.hcvguidelines.org. Accessed May 3, 2016.

14. Ghany MG, Nelson DR, Strader DB, et al. An update on treatment of genotype 1 chronic hepatitis $\mathrm{C}$ virus infection: 2011 practice guideline by the American Association for the Study of Liver Diseases. Hepatology 2011;54:1433-44.

15. US National Institutes of Health. Clinicaltrials.gov Web Site. US National Institutes of Health Web Site. http://clinicaltrials.gov/. Accessed May 3, 2016. 